\title{
Forebrain ischemia produces hippocampal damage and a persistent working memory deficit in rats
}

\author{
PAUL J. COLOMBO, HASKER P. DAVIS, NEIL SIMOLKE, and FRANK MARKLEY \\ University of Colorado, Colorado Springs, Colorado
}

and

\author{
BRUCE T. VOLPE \\ Cornell University Medical College, New York, New York
}

\begin{abstract}
Rats subjected to transient forebrain ischemia (postischemic, or PI, rats) were given extensive postoperative training (136 trials) on a radial eight-arm maze to determine whether working memory was permanently impaired. Animals were pretrained to an asymptote performance. After ischemia, PI rats demonstrated a permanent working memory impairment $(p<.05)$. Transient forebrain ischemia appears to preferentially damage hippocampal CA1 neurons. These findings provide further evidence that PI rats demonstrate morphologic and behavioral sequelae similar to those of some human survivors of cardiac arrest.
\end{abstract}

Rats with ischemic hippocampal damage induced by four-vessel occlusion demonstrate the morphologic and behavioral deficits observed in some survivors of cardiac arrest. In particular, rats subjected to $30 \mathrm{~min}$ of forebrain ischemia show severe neuronal damage in the CA1 region of the hippocampus and a behavioral deficit in working memory performance on the radial arm maze (Davis, Baranowski, Pulsinelli, \& Volpe, 1987; Davis, Tribuna, Pulsinelli, \& Volpe, 1986; Volpe, Pulsinelli, \& Davis, 1985; Volpe, Pulsinelli, Tribuna, \& Davis, 1984). Postmortem examination of humans with a circumscribed amnesic syndrome after cardiac arrest has revealed a loss of neurons in the CA1 region of the hippocampus (Volpe \& Petito, 1985; Zola-Morgan, Squire, \& Amaral, 1986). During antemortem examination, cardiac arrest survivors have demonstrated impaired acquisition of new and variable information (Volpe \& Hirst, 1983; Zola-Morgan et al., 1986).

Previously, it has been demonstrated that although postischemic (PI) animals are initially impaired on the working memory component of a radial maze task, recovery of working performance may occur as postoperative training continues (Davis et al., 1987). Task difficulty and the amount of behavioral training prior to ischemia are two factors that may influence memory performance. Animals given extensive pretraining on a radial arm maze in which five of eight arms were baited were able to improve their working memory performance if they had received extensive behavioral training prior to ischemic

This research was supported by USPHS Grant MH40090-02 and American Heart Association Grant 851098. Address correspondence to Hasker P. Davis, Department of Psychology, University of Colorado, Colorado Springs, CO 80933. insult (Davis et al., 1987). PI rats that had not received pretraining remained impaired on working memory performance (Davis et al., 1986; Volpe et al., 1984; Volpe et al., 1985). The present study addressed the role of task difficulty in the recovery of working memory performance after ischemic injury. Extensively pretrained animals were tested after increasing the difficulty of the working performance component of an eight-arm radial arm maze by baiting all eight arms.

\section{METHOD}

\section{Subjects}

Male Wistar rats (Hilltop, PA) weighing between 200 and $300 \mathrm{~g}$ served as subjects. The animals were individually housed in polyurethane cages $(33 \times 21 \times 19 \mathrm{~cm})$ in a temperature-controlled $\left(21^{\circ} \mathrm{C}\right)$ facility and maintained on a partial deprivation feeding schedule with ad-lib access to water. The rats were on a 12:12 light:dark schedule (6 a.m. -6 p.m.) and all animals were tested between 7 a.m. and 2 p.m.

\section{Surgical Procedure}

The method for subjecting rats to forebrain ischemia has been described in detail previously (Pulsinelli \& Brierly, 1979). Briefly, on Day 1 the animals were anesthetized by inhalation of $2.0 \%$ halothane. The vertebral arteries were occluded at the first cervical vertebra by electrocautery, and silastic ligatures were placed around each carotid artery without interruption of blood flow. On Day 2 forebrain ischemia was produced in the animals by tightening the carotid artery ligatures for $30 \mathrm{~min}$. Rats that lose their righting response within $1 \mathrm{~min}$ of carotid clamping and for the subsequent $30 \mathrm{~min}$ have been shown to have cerebral blood flow decreased to less than $10 \%$ of the blood flow of control animals (Pulsinelli, Levy, \& Duffy, 1982). The control animals were subjected to anesthesia and carotid manipulation. After 30 days of recovery, no detectable physical, feeding, or locomotor differences between operated and control animals were observed.

Behavioral Apparatus and Procedure

The rats were tested on an eight-arm radial maze. Each arm $(10 \times 60 \mathrm{~cm})$ had clear Plexiglas side walls $(28.5 \mathrm{~cm}$ high $)$ and a recessed 
food hole $(3.4 \mathrm{~cm}$ in diameter) at the end. Maze arms were positioned at equal intervals around a central platform $(57 \mathrm{~cm}$ in diameter). The room was rich in stationary extramaze cues. After an initial 3-day acclimation, the animals were placed on a partial food deprivation schedule and maintained at approximately $80 \%$ of their ad-lib weights. During a 3-day adaptation, individual animals were allowed to explore the maze for $10 \mathrm{~min}$ with reward pellets (Bio Serv, Frenchtown, NJ, $190 \mathrm{mg}$ ) scattered throughout the maze. Following adaptation, training was begun and continued in a consistent manner throughout the experiment. Single reward pellets were placed in each of the eight arms. An individual animal was placed in the center of the maze and was allowed to choose arms until all eight pellets had been consumed, 16 choices had been made, or $600 \mathrm{sec}$ had elapsed. The animals were confined to the center of the maze by guillotine doors for $3 \mathrm{sec}$ between arm choices. The initial entry into an arm was a correct choice, and any subsequent entry into a previously entered arm was a working error. The animals were given 55 daily preoperative trials.

One day after Trial 55, the animals were subjected to ischemia, allowed 30 postoperative recovery days, and then returned to maze training for an additional 136 daily trials. All rats were assigned code numbers at the time of surgery, so that behavioral training was blind.

\section{Histology}

Following behavioral training, the animals were perfused with a solution of formaldehyde, glacial acetic acid, and methanol (1:1:8), and brain sections $(7 \mu)$ were taken at several levels of the hippocampus (approximately bregma -3.3 to $-5.3 \mathrm{~mm}$ ). Sections were stained with hematoxylin and eosin. Without knowledge of the experimental conditions, one of us qualitatively assessed the neuronal damage on a scale of 0-3 with $0=$ normal brain, $1=a$ few neurons damaged, $2=$ many neurons damaged, 3 = majority of neurons damaged.

\section{RESULTS}

\section{Preoperative Behavior}

A multivariate analysis of variance (MANOVA) with repeated measures, using condition and trials as independent variables, was performed on the last 10 preoperative trials. No significant difference between control and PI rats was detected on working memory performance $[F(1,20) \leq 1.0, p>.05]$. There was neither a significant effect of trials $[F(9,180)=1.27, p>.05]$ nor a significant interaction between trial and condition $[F(9,180) \leq 1.0, p>.05]$. Thus, PI rats had reached asymptotic preoperative performance.

\section{Postoperative Behavior}

Individual trials were averaged into blocks of eight trials, and then a MANOVA with repeated measures, using condition and trials as independent variables, was performed. The mean numbers of working errors for the 136 postoperative trials are shown for blocks of 8 trials in Figure 1. Both control $(N=11)$ and PI $(N=11)$ rats demonstrated improved working performance on postoperative trials $[F(16,320)=6.29, p<.001]$. However, PI animals made significantly more errors than did control animals $[F(1,20)=5.13, p<.05]$, and there was no trial $\times$ condition interaction $[F(16,320) \leq 1.0$, $p>.05]$.

A MANOVA was performed on the last 64 trials to determine whether the memory deficit in PI rats persisted after the animals had reached asymptotic performance (see Figure 1). There was no significant effect of trials $[F(7,140)=1.47, p>.05]$ or interaction between condition and trial $[F(7,140) \leq 1.0, p>.05]$ during these 64 trials. However, PI animals continued to make significantly more errors than did control animals $[F(1,20)=4.47$, $p<.05]$. Thus, although PI animals demonstrated an initial improvement in postoperative working memory performance, they remained impaired, compared with control animals, even when allowed to reach optimal performance.

WORKING MEMORY PERFORMANCE ON AN 8-ARM RADIAL MAZE

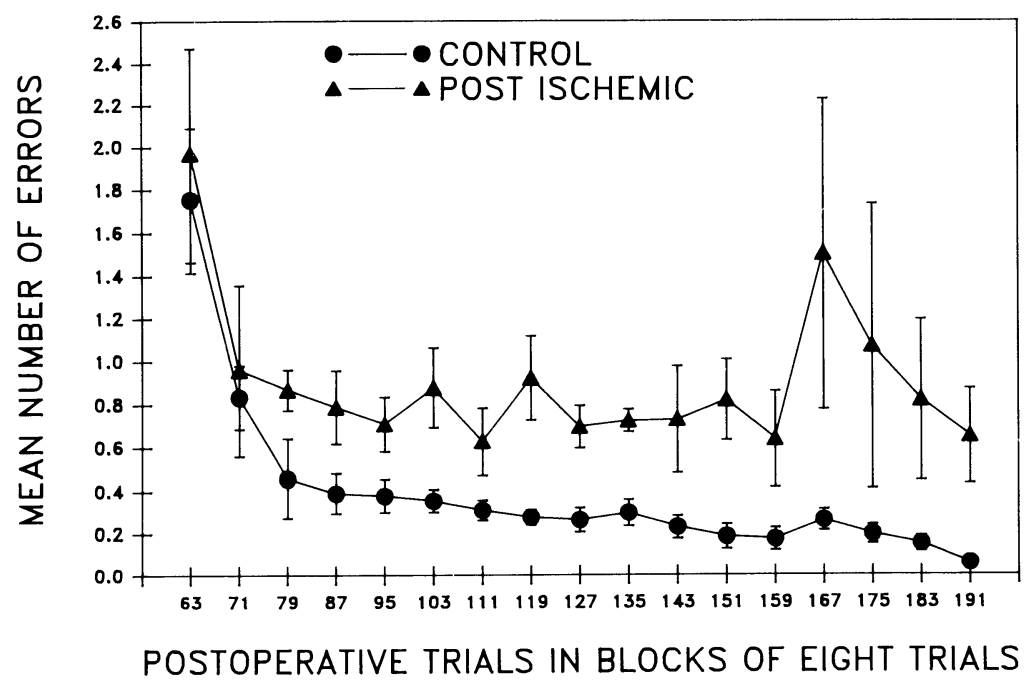

Figure 1. Mean postoperative working errors $( \pm S E M)$ for postischemic $(N=11)$ and control $(N=11)$ rats in blocks of eight trials. 
Table 1

Severity and Distribution of Ischemic Brain Damage in Postischemic Rats $(N=11)$

\begin{tabular}{|c|c|c|}
\hline \multicolumn{3}{|l|}{ Neocortex } \\
\hline Anterior & .5 & \pm .79 \\
\hline Posterior & & 0 \\
\hline \multicolumn{3}{|c|}{ Anterior Hippocampus } \\
\hline CA1 & 2.63 & \pm .65 \\
\hline CA2 & & 0 \\
\hline CA3 & & 0 \\
\hline \multicolumn{3}{|c|}{ Posterior Hippocampus } \\
\hline \multicolumn{3}{|c|}{ Dorsal Anterior } \\
\hline Subiculum & 1.6 & \pm 1.30 \\
\hline CA1 & 2.1 & \pm .93 \\
\hline CA2 & & 0 \\
\hline CA3 & & 0 \\
\hline Striatum & 2.13 & \pm 1.03 \\
\hline Thalamus & .41 & \pm .66 \\
\hline
\end{tabular}

Note-No damage was observed in any of these areas in the control group $(N=11)$.

\section{Histology}

The extent and distribution of neuronal damage at the plane of the anterior hippocampus (bregma $-3.3 \mathrm{~mm}$ ) to the posterior hippocampus (bregma $-5.3 \mathrm{~mm}$ ) is shown in Table 1. The most extensive neuronal loss was in the CA1 field of the hippocampus, followed by striatum, posterior CA1 of hippocampus, and dorsal subiculum.

\section{DISCUSSION}

In the past, extensive pretraining of ischemic animals may have contributed to the recovery of working memory performance on a radial arm maze with five of eight arms baited. The present data demonstrate that PI animals had a persistent working memory deficit when the task difficulty was increased. Extensive preoperative training did not significantly reduce the working memory performance deficit. Further systematic variations of task difficulty and amount of pretraining will be necessary to determine the precise effects of these two variables on working memory performance in PI rats.

Transient forebrain ischemia probably causes neuronal injury outside the CA1 regions of the hippocampus. Although some of this injury is reversible (Petito \& Pulsinelli, 1984), neuronal loss in the subiculum and dorsal lateral caudate raises the question of the anatomical distribution of damage responsible for impaired working memory performance. In previous experiments, rats with radiofrequency lesions of the striatum were not impaired on the working component of a radial arm maze with 7 of 12 arms baited (Volpe, Pulsinelli, Simolke, \& Davis, 1986). Furthermore, animals with ibotenic acid lesions in the subiculum or dorsolateral caudate did not show impairment of goal arm choices (working memory) on a modified T-maze using the delayed nonmatching to sample paradigm (Volpe, Waczek, Colombo, \& Davis, 1987).
PI rats were impaired on both the radial maze and modified T-maze tasks. Furthermore, subiculum or dorsolateral caudate injury alone has not been associated with working memory deficits in PI rats. It may be that hippocampal ischemic injury, in particular the widespread CA1 injury, is sufficient to cause a working memory deficit in PI animals.

The PI rat models the morphologic damage and behavioral deficits observed in some survivors of cardiac arrest. Specifically, PI rats have extensive damage in the $\mathrm{CA} 1$ region of the hippocampus and show impaired learning and memory of new information. The present study indicates that the memory impairment in PI rats with hippocampal damage can be permanent.

\section{REFERENCES}

Davis, H. P., Baranowski, J. R., Pulsinelli, W. A., \& Volpe, B. T. (1987). Retention of reference memory following ischemic hippocampal damage. Physiology \& Behavior, 39, 783-786.

Davis, H. P., Tribuna, J., Pulsinelli, W. A., \& Volpe, B. T. (1986). Reference and working memory of rats following hippocampal damage induced by transient forebrain ischemia. Physiology \& Behavior, 37, 387-392.

Petito, C. K., \& Pulsinelli, W. A. (1984). Delayed neuronal recovery and neuronal death in rat hippocampus following severe cerebral ischemia: Possible relationship to abnormalities in neuronal processes. Journal of Cerebral Blood Flow \& Metabolism, 4, 194-205.

Pulsinelli, W. A., \& Brierly, J. B. (1979). A new model of bilateral hemispheric ischemia in the unanesthetized rat. Stroke, 10, 267-272.

Pulsinelli, W. A., Levy, D. E., \& DufFy, T. E. (1982). Regional cerebral blood flow and glucose metabolism following transient forebrain ischemia. Annals of Neurology, 11, 499-509.

VolPE, B. T. , \& HIRST, W. (1983). The characterization of an amnesic syndrome following hypoxic ischemic injury. Archives of Neurology, 40, 436-440.

VolPe, B. T., \& Petrto, C. K. (1985). Dementia with bilateral medial temporal lobe ischemia. Neurology, 35, 1793-1797.

Volpe, B. T., Pulsinelli, W. A., \& Davis, H. P. (1985). Amnesia in humans and animals after ischemic cerebral injury. Annals of the New York Academy of Science, 444, 492-493.

Volpe, B. T., Pulsinelli, W. A., Simolke, N. E., \& Davis, H. P. (1986). Rats with ischemic induced hippocampal injury demonstrate dissociated memory impairment on a 12-arm radial maze. Annals of Neurology, 20, 154.

Volpe, B. T., Pulsinelli, W. A., Tribuna, J., \& Davis, H. P. (1984). Behavioral performance of rats following transient forebrain ischemia. Stroke, 15, 558-562.

VolPe, B. T., WACZeK, B., Colombo, P. J., \& DAVIS, H. P. (1987). Ischemic induced hippocampal injury in rats causes dissociated memory impairment on a modified T-maze. Society for Neuroscience Abstracts, 13, 802.

Zola-Morgan, S., Souire, L. R., \& Amaral, D. G. (1986). Human amnesia and the medial temporal region: Enduring memory impairment following a bilateral lesion limited to field CA1 of the hippocampus. Journal of Neuroscience, 6, 2950-2967.

(Manuscript received for publication November 12, 1987.) 